\title{
HANDWRITTEN RECOGNITION BY USING MACHINE LEARNING APPROACH
}

\author{
P.Thangamariappan \\ M.E., Computer Science and Engineering \\ Government College of Technology \\ Coimbatore -641 013
}

\begin{abstract}
Deep learning is the most powerful machine learning technique in current generation. This paper presents the result of handwritten recognition using deep learning. Handwriting is unique to each individual. So the handwriting is differed from one person to another person. Handwritten Recognition can be done in two ways. One is Online Handwritten recognition and another one is Offline Handwritten Recognition. Online Handwritten recognition system, which takes the input at run time and Offline Handwritten Recognition which works on scanned images. Offline handwritten is the hardest to find the handwriting. MNIST data set is used for this handwritten digit recognition process and it has 70000 handwritten digits. Many Machine Learning Algorithms are developed which can be used for this digit classification. This paper performs the analysis of accuracies and performance measures of algorithm Convolutional Neural Networks (CNN). The proposed approach recognizes with overall accuracy is $93 \%$.
\end{abstract}

Keywords: Handwritten Recognition, MNIST Dataset, Deep Learning, Convolutional Neural Networks (CNN).

\section{INTRODUCTION}

Handwritten Recognition (HR) is a challenging task in the field of pattern recognition. Because every person in this world has their own way of writing. In real-time applications like the conversion of handwritten information into digital format, verification of signatures, number plate recognition, this kind recognition is required. This recognition was implemented using many Machine Learning techniques like Random Forest, Naive Bayes and Support Vector Machine, Convolution Neural Network(CNN), etc. The accuracy and correctness are mostly crucial in handwritten digit recognition applications. Even 1\% error may lead to inappropriate results in real-time applications.

The MNIST (Modified National Institute of Standards and Technology database) data set is used for this recognition process. The MNIST data set has 70,000 handwritten digits. Here 60,000 images are

\author{
Dr.J.C.Miraclin Joyce Pamila \\ M.E., PhD, Computer Science and Engineering \\ Government College of Technology \\ Coimbatore -641 013
}

used training examples and 10,000 images are used testing examples. MNIST database is a combination of Special Database 1 and Special Database 3. Special Database1 consists of digits written by school students. Special Database 3 consists of digits written by employees. Half of the training and test set have taken from NIST's training dataset, and the remaining training set and test set were taken from NIST's testing dataset.

\section{THE PROPOSED RECOGNITION SYSTEM}

\section{A. DEEP LEARNING}

Deep Learning is also known as Deep Structured learning, Deep Machine Learning, Hierarchical learning. DEEP LEARNING - The word "DEEP" is refers to the number of layers through which the data is transformed. Deep Learning is the combination of machine learning and Artificial Neural Network (ANN). The learning can be supervised, unsupervised and semi-supervised. Deep learning architectures are Deep Neural network(DNN), Deep Belief Network (DBN), Recurrent neural Network(RNN) and Convolutional Neural Networks(CNN) have been applied to fields including speech recognition, natural language processing(NLP), Audio Recognition. Deep learning algorithms are applied to unsupervised learning tasks in machine learning. This is an important benefit because un labeled data are more abundant than the labelled data.

\section{B. CONVOLUTIONAL NEURAL NETWORK $(\mathrm{CNN})$}

A convolutional neural network is a combination of input and an output layer, as well as multiple hidden layers. The hidden layers of a CNN typically consist of a series of convolutional layers that convolve with a dot product.

The activation function is called a RELU layer. CNN is regularized versions of multilayer perceptrons (MLP). Multilayer perceptrons usually called fully connected networks, that is, each neuron in one layer is connected to all neurons in the next layer. 


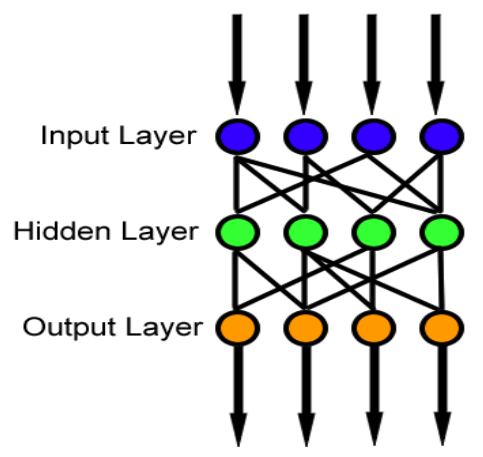

\section{IMPLEMENTATION}

Tensorflow

TensorFlow is an amazing data stream in machine learning library made by the Brain Team of Google and made open source in the year 2015. It is intended to ease the use and broadly relevant to both numeric and neural system issues in different spaces. TensorFlow is a low- level tool for doing entangled math and it targets specialists who recognize what they're doing to construct the exploratory learning structures, to play around and to transform them into running programs. Mostly, it can be considered as a programming framework in which one can perform the calculations as graphs. Nodes in the graph says the math activities and the edges contains the multi-dimensional information clusters (tensors) which is related among them.

Anaconda3 5.3.1

Anaconda is a free and open-source appropriation of the Python and $\mathrm{R}$ programming for logical figuring like information science, AI applications, large-scale information preparing, prescient investigation, and so forth. Anaconda accompanies in excess of 1,400 packages just as the Conda package and virtual environment director, called Anaconda Navigator, so it takes out the need to figure out how to introduce every library freely. Anaconda Navigator is a Graphical User Interface (GUI) incorporated into Anaconda appropriation, it enable the clients to dispatch applications and overview the conda packages, conditions, channels without utilize the command- line directions.

\section{Python 3.7}

Python is broadly utilized universally and is a high-level programming language. It was primarily introduced for prominence on code, and its language structure enables software engineers to express ideas in fewer lines of code. Python is a programming language that gives you a chance to work rapidly and coordinate frameworks more effectively.

\section{A. STRUCTURE OF THE MODEL}

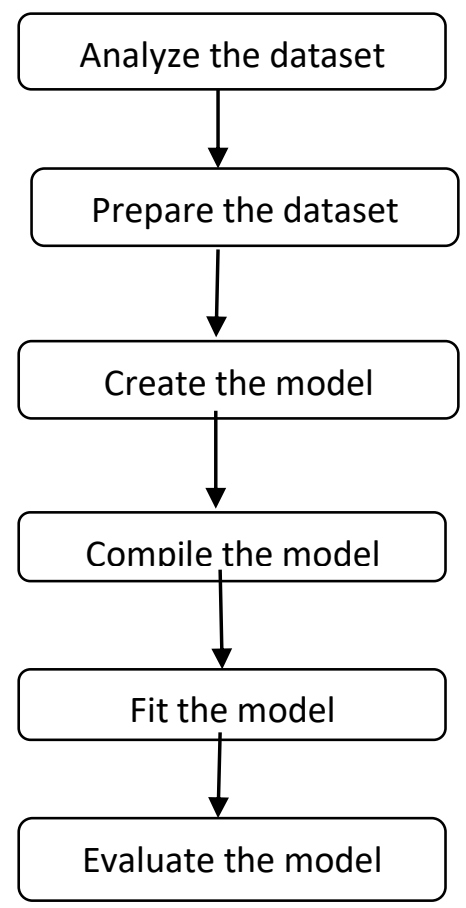

\section{B. ANALYSE THE DATASET}

This paper is used in MNIST (Mixed National Institute of Standards and Technology database) dataset. This dataset is created by Yaan LeCun, Corinna Cortes, Christopher Burges. The image size is $28 \times 28$ pixel square. So the total of 784 pixels. Here the 60000 images are used in training images and 10000 images are used in testing images. Also the MNIST dataset top error rate is 0.21 . This error rate is achieved by Convolutional Neural Network.

\section{PREPARE THE DATASET}

Initially loading the MNIST dataset by using mnist function in Keras. It returns two tuples. First tuple holding the train data and train label and another one tuple holding the test data and test label. After loading the dataset, we need to analyze and pre-process the dataset.

Reshaping - This is needed step. Because, in Deep Learning, we provide the original data and label, then that each image has the dimension of [28x28]. So, we will get $28 \times 28=784$ pixel intensities.

Data type - After reshaping, need to change the pixel intensities to float 32 datatype. As grayscale image pixel intensities are ranged from 0 to 255 . 
Normalize - After declare the datatype we need normalize the floating point values in the range of $0-1$. The normalize step is to improve computational efficiency.

\section{ONE HOT ENCODING}

A one hot encoding is used to represent the categorical variables. The categorical variables are represented as binary vectors. First, it requires that the categorical values are mapped to integer values. Then, each integer value is replaced with one and others are zero.

\section{CREATE THE MODEL}

Here using a simple Multi-Layer Perceptron (MLP) as our neural network model. Also the neural network model is used in 784 input neurons. Two hidden layers are used. The 512 neurons in hidden layer 1 and 256 neurons in hidden layer 2.ReLU is used as the activation function for the hidden layers and softmax is used as the activation function for the output layer.

\section{E. COMPILE THE MODEL}

After create the model, we need to compile the model for optimization and learning. We will use multi-label classification problem for the loss function.

\section{F. FIT THE MODEL}

After compile the model, we need to fit the model with the help of MNIST dataset.

\section{G. EVALUATE THE MODEL}

After fit the model, the model can be evaluated on the unseen test data. Matplotlib is used to visualize how our model reacts at different epochs on both training and testing data.

\section{EXPERIMENTAL RESULT}

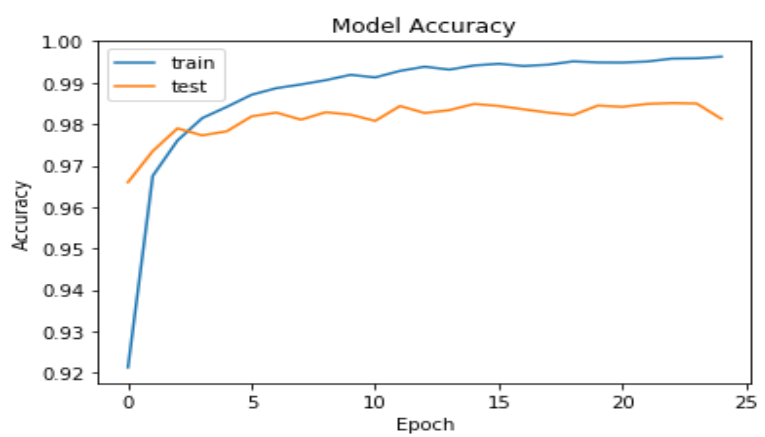

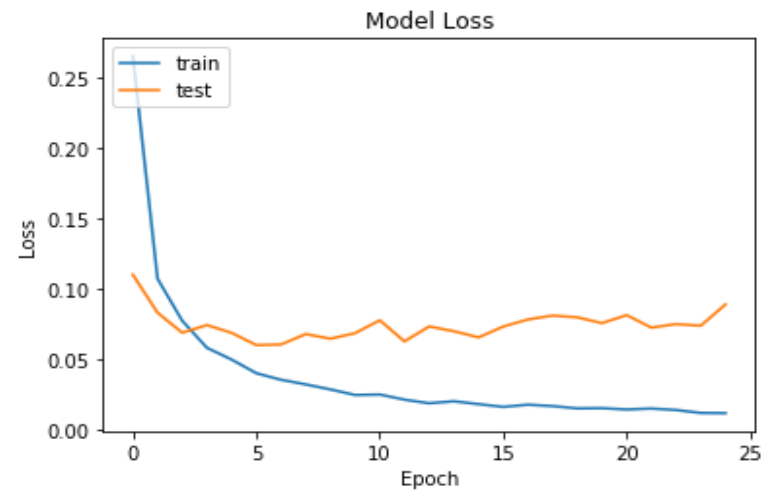

Fig. 1 Model accuracy and Model loss for test and training dataset.

\begin{tabular}{|l|l|l|}
\hline & DATASET & VALUE \\
\hline TEST & MNIST & 88.3 \\
SCORE & DATASET & \\
\hline TEST & MNIST & 98.5 \\
ACCURACY & DATASET & \\
\hline
\end{tabular}

Table: 1Comparison of accuracy algorithms

From the Table we observe the best accuracy rate of $98.5 \%$ is provided by Multi Layer Perceptron.

\section{CONCLUSION}

The result of our work shows that the maximum accuracy $98.5 \%$ was obtained in MNIST dataset using the multilayer perceptron neural network technique. A Multilayer Perceptron Neural Network based method is presented here for increasing accuracy of offline handwritten text recognition. We have tested our developed algorithm. The result is shown below as output image . Future works is focused on further develop the accuracy of recognition by improving preprocessing of data which is fed into deep convolution neural network.
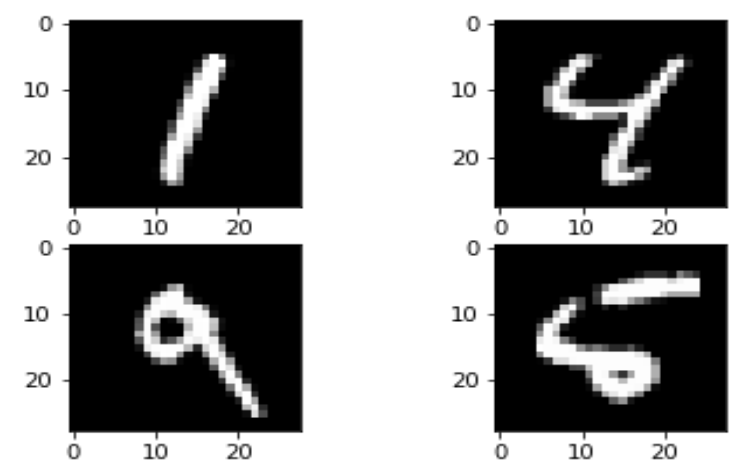

Fig. 2 Handwritten Digits 


\section{International Journal of Engineering Applied Sciences and Technology, 2020 Vol. 4, Issue 11, ISSN No. 2455-2143, Pages 564-567 \\ Published Online March 2020 in IJEAST (http://www.ijeast.com)}

\section{REFERENCES}

[1] Cireşan, D. C., Meier, U., Gambardella, L. M., \& Schmidhuber, J. (2010). Deep, big, simple neural nets for handwritten digit recognition. Neural computation, 22(12), 3207-3220.

[2] Byun, H., \& Lee, S. W. (2003). A survey on pattern recognition applications of support vector machines. International Journal of Pattern Recognition and Artificial Intelligence, 17(03), 459-486.

[3] Deng, L. (2012). The mnist database of handwritten digit images for machine learning research [best of the web]. IEEE Signal Processing Magazine, 29(6), 141142.

[4] Purohit, A., \& Chauhan, S.S. (2016). A Literature Survey on Handwritten Character Recognition. (IJCSIT) International Journal of Computer Science and Information Technologies, 7(1), 1-5.

[5] Singh, V., \& Lal, S. P. (2014, November). Digit recognition using single layer neural network with principal component analysis. In Asia-Pacific World Congress on Computer Science and Engineering (pp. 1-7). IEEE.

[6] Yadav, P., \& Yadav,N. (2015). Handwriting recognition system- A review. International Journal of Computer Applications, 114(19), $36-40$.

[7] Bottou, L., Cortes, C., Denker, J. S., Drucker, H., Guyon, I., Jackel, L. D., ... \& Vapnik, V. (1994, October). Comparison of classifier methods: a case study in handwritten digit recognition. In Proceedings of the 12th IAPR International Conference on Pattern Recognition, Vol. 3-Conference C: Signal Processing (Cat. No. 94CH3440-5) (Vol. 2, pp. 77-82). IEEE.

[8] Ghosh, M. M. A., \& Maghari, A. Y. (2017, October). A comparative study on handwriting digit recognition using neural networks. In 2017 International Conference on Promising Electronic Technologies (ICPET) (pp. 77-81). IEEE.

[9] Xiao, X., Jin, L., Yang, Y., Yang, W., Sun, J., \& Chang, T. (2017). Building fast and compact convolutional neural networks for offline handwritten Chinese character recognition. Pattern Recognition, 72, 72-81.

[10] Zhang, X. Y., Bengio, Y., \& Liu, C. L. (2017). Online and offline handwritten chinese character recognition: A comprehensive study and new benchmark. Pattern Recognition, 61, 348360 .

[11] Larasati, R., \& KeungLam, H. (2017, November). Handwritten digits recognition using ensemble neural networks and ensemble decision tree. In 2017 International Conference on Smart Cities, Automation \& Intelligent Computing Systems (ICON-SONICS) (pp. 99-104). IEEE.

[12] Krizhevsky, A., Sutskever, I., \& Hinton, G. E. (2012). Imagenet classification with deep convolutional neural networks. In Advances in neural information processing systems (pp. 1097-1105).

[13] Simonyan, K., \& Zisserman, A. (2014).Very deep convolutional networks for largescale image recognition. arXiv preprint arXiv:1409.1556.

[14] Boureau, Y. L., Ponce, J., \& LeCun, Y. (2010). A theoretical analysis of feature pooling in visual recognition. In Proceedings of the 27th international conference on machine learning (ICML10) (pp. 111-118).

[15] Rui, T., Shen, C. L., Ding, J., \& Zhang, J. L. (2005). Handwritten character recognition using principal component analysis. MINIMICRO SYSTEMS, 26, 289-292.

[16] Kaensar, C. (2013). A comparative study on handwriting digit recognition classifier using neural network, support vector machine and k-nearest neighbor. In The 9th International Conference on Computing \& Information Technology (IC2IT2013) (pp. 155-163). Springer, Berlin, Heidelberg.

[17] Pendlebury, J., Xiong, H., \& Walshe, R. (2012, October). Artificial neural network simulation on CUDA. In 2012 IEEE/ACM 16th International Symposium on Distributed Simulation and Real Time Applications (pp. 228-233). IEEE. 